\title{
A simple explanation of the linear rms-mean flux relation in accreting objects
}

\author{
C. Koen

\begin{abstract}
Department of Statistics, University of the Western Cape, Private Bag X17, Bellville, 7535 Cape, South Africa e-mail: ckoen@uwc.ac.za
\end{abstract}

Received 6 July 2016 / Accepted 4 September 2016

\begin{abstract}
Aims. We provide an alternative to existing theories for the origin of linear rms-mean flux relations observed in a variety of accreting systems, from AGN to white dwarfs.

Methods. Standard statistical theory and simulations are used to explore the possibility that the relations are due to simple scaling effects.

Results. The proposed theory can reproduce the unit slope of the rms-mean flux relation observed in the most extensive study published to date. The close similarity of the fractional variability in different objects deserves further study.
\end{abstract}

Key words. accretion, accretion disks - methods: statistical

\section{Introduction}

A number of papers have drawn attention to linear relations between the mean flux level and the root mean square (rms) of flux variations, as observed in a variety of accreting astronomical objects. Some of the relevant papers are Uttley \& McHardy (2001; active galactic nuclei and a millisecond pulsar), Gleissner et al. (2004; a black hole), Van de Sande et al. (2015; white dwarfs), and Zamanov et al. (2016; white dwarfs and symbiotic stars). Further references are available in these sources.

Uttley et al. (2005) proposed an explanatory model for these linear rms-mean relations. Key ingredients are non-linearity of the light curves, and lognormal flux distributions. An alternative interpretation of the relations is presented below in terms of simple statistical identities. No specific distributional assumptions or forms of the time series are required.

\section{Statistical model}

Understanding the model is facilitated by thinking of the light curve of the accreting system as the manifestation of a stochastic process, consisting of a succession of values of a random variable $X$. The random process has mean

$E X=\mu$,

where $E$ is the expectation (ensemble mean) operator, while the standard deviation (rms) of its variations is given by

$\sigma=\sqrt{\operatorname{var}(X)}=\sqrt{E(X-E X)^{2}}$.

Another useful statistic is the coefficient of variation, defined as

$C V_{0}=\sigma / \mu$.

First we consider the simple situation where the process switches to a new mean level. If the flux increases by a factor $\beta$, so that
$X \rightarrow \beta X$, then the following simple standard statistical relations hold:

$E(\beta X)=\beta \mu$

$\operatorname{var}(X)=E[(\beta X)-E(\beta X)]^{2}$

$=\beta^{2} \sigma^{2}$
$C V=\sqrt{\beta^{2} \sigma^{2}} /(\beta \mu) \equiv C V_{0}$.

Obviously then, if $X$ switches between any number of welldefined mean levels, the coefficient of variation (or fractional variability) remains constant at $C V_{0}$.

For an illustrative example of what happens if the mean level is continuously variable, the reader is referred to the simulated dataset plotted in Fig. 1. Here,

$X(t)=g(t) z(t)$,

where $g(t)$ is a smooth trend (an integrated random walk in this example), and $z(t)$ is Gaussian with mean 20 and standard deviation 1 (arbitrary illustrative values).

The series in Fig. 1 is then subdivided into bins, and the mean and standard deviations of the points $X(t)$ in each bin calculated. The results are plotted in Fig. 2 for a few different bin widths (or timescales). For comparison, the lines

$s_{X}=\frac{1}{20} \bar{X}$

are also plotted; they correspond to the constant coefficient of variation $C V=0.05$. Clearly the expected rms-mean relation is obtained, regardless of the choice of bin width.

Figure 3 shows a second simulated dataset, again with an integrated random walk as the trend function. The model for the random process is more realistic: instead of white noise, $z(t)$ in (2) is taken to be an autoregressive (AR) series

$z(t)=10+u(t)$
$u(t)=\alpha u(t-1)+\epsilon(t)$, 


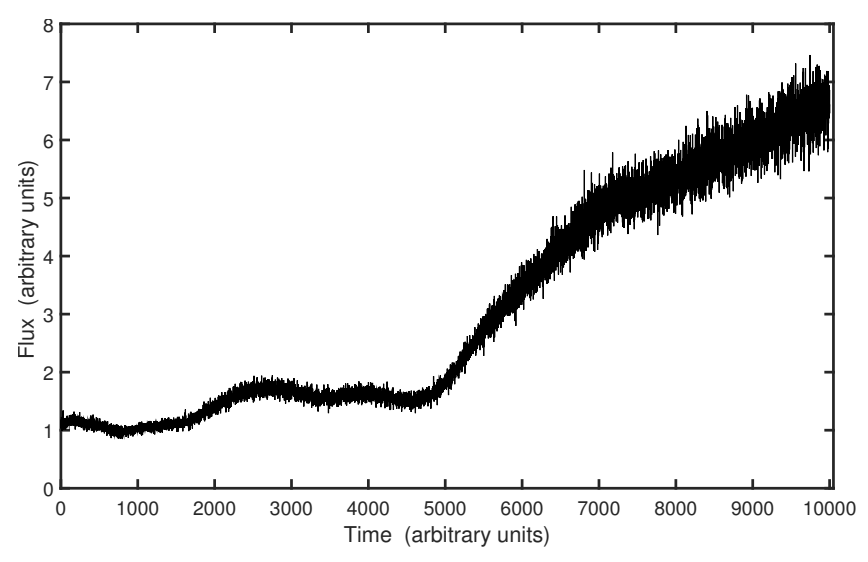

Fig. 1. A simulated dataset $X(t)$, consisting of Gaussian white noise $z(t)$ multiplied by a smooth trend function $g(t)$.

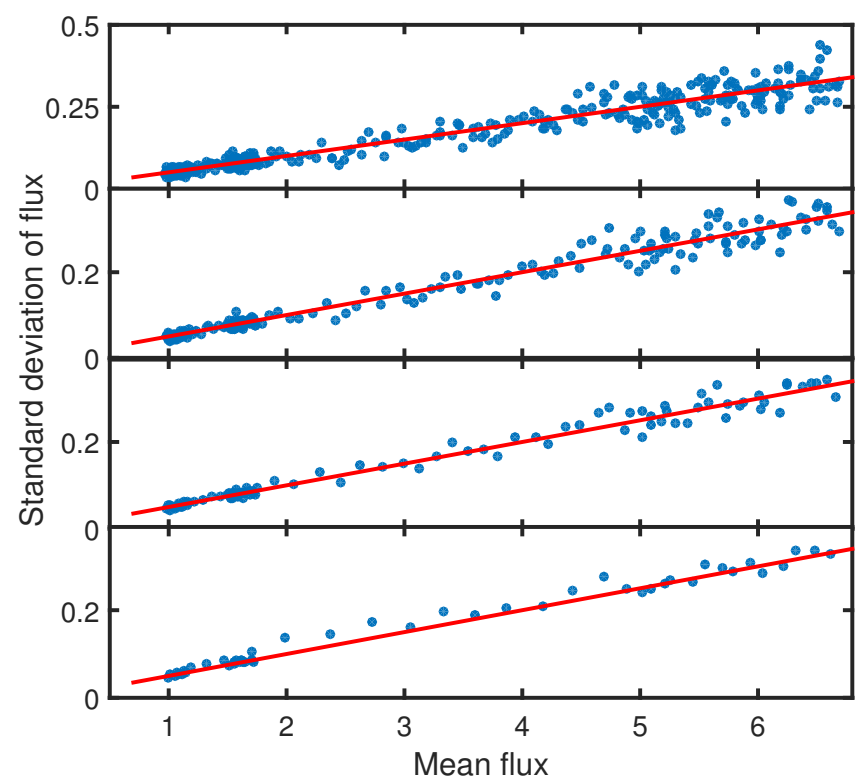

Fig. 2. Relation between the MSE and the mean for the light curve in Fig. 1. The simulated data were subdivided into bins, and the means and standard deviations of the values in each bin calculated. Bin widths, from top to bottom, are $25,50,100$, and 200 points. The straight lines correspond to constant coefficients of variation of 0.05 .

where $\alpha$ is a constant (equal to 0.9 in Fig. 3), and $\epsilon(t)$ is white noise with standard deviation $\sigma_{\epsilon}=0.1$. The variance of $z(t)$ in this case is

$\operatorname{var}(z)=\sigma_{z}^{2}=\frac{\sigma_{\epsilon}^{2}}{1-\alpha^{2}}$

(e.g. Chatfield 2003). It follows that $\sigma_{z}=0.23$ and $C V=0.023$.

The equivalent of Fig. 2 is plotted in Fig. 4, which is based on the data in Fig. 3. Comparison with Fig. 2 shows that although linear rms-mean flux relations persist, the exact form is now dependent on the bin size. In particular, for large bin sizes the expected forms

$s_{X}=0.023 \bar{X}$

are recovered, whereas for small bins the slopes systematically decrease with bin size.

The explanation of the dependence on bin size (or timescale) lies in the form of the variability of the adopted AR time series -

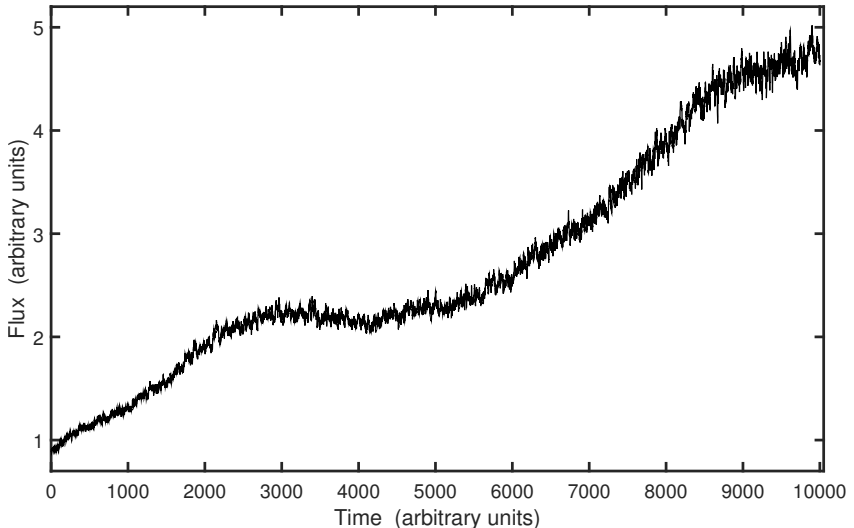

Fig. 3. A simulated dataset $X(t)$, consisting of a first-order autoregressive time series $z(t)$ multiplied by a smooth trend function $g(t)$.

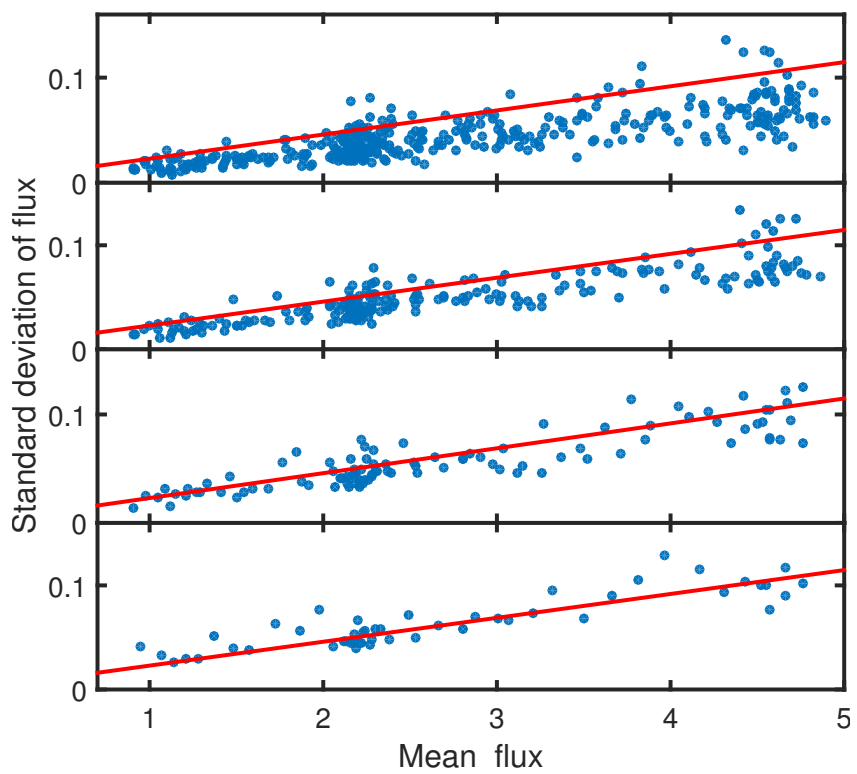

Fig. 4. Relation between the MSE and the mean for the light curve in Fig. 3. The simulated data were subdivided into bins, and the means and standard deviations of the values in each bin calculated. Bin widths, from top to bottom, are 25, 50,100, and 200 points. The straight lines correspond to constant coefficients of variation of 0.023 .

see Fig. 5 for three realisations. It is clear that as $\alpha$ increases, sustained deviations from the mean also increase; which explains why $\sigma_{z}$ increases with $\alpha$ in (3). Since the "coherence time" of these deviations increases with $\alpha$, longer sections of the time series are needed to plumb the full extent of the deviations from the mean, i.e. the variance of the series. Conversely, if short stretches of the time series (i.e. small bin sizes) are used to estimate $\sigma_{X}^{2}$, the variance will be underestimated.

In the frequency domain the power spectral density (PSD) is of interest: at frequency $\omega$ it is defined as

$$
\begin{aligned}
& f_{X}(\omega)=c \sum_{k=-\infty}^{\infty} \gamma_{X}(k) \mathrm{e}^{-\mathrm{i} \omega k} \\
& \gamma_{X}(k)=E[X(t)-E X(t)][X(t+k)-E X(t+k)],
\end{aligned}
$$

where $c$ is a normalising constant and $\gamma_{X}$ is the autocovariance function of the series $X$. If $X$ is scaled by $\beta$, the PSD is scaled by $\beta^{2}$ at all frequencies - in other words, the scaling holds at all timescales (as is evident especially from Fig. 2). In this regard, see also Uttley \& McHardy (2001), who note that the amplitudes 


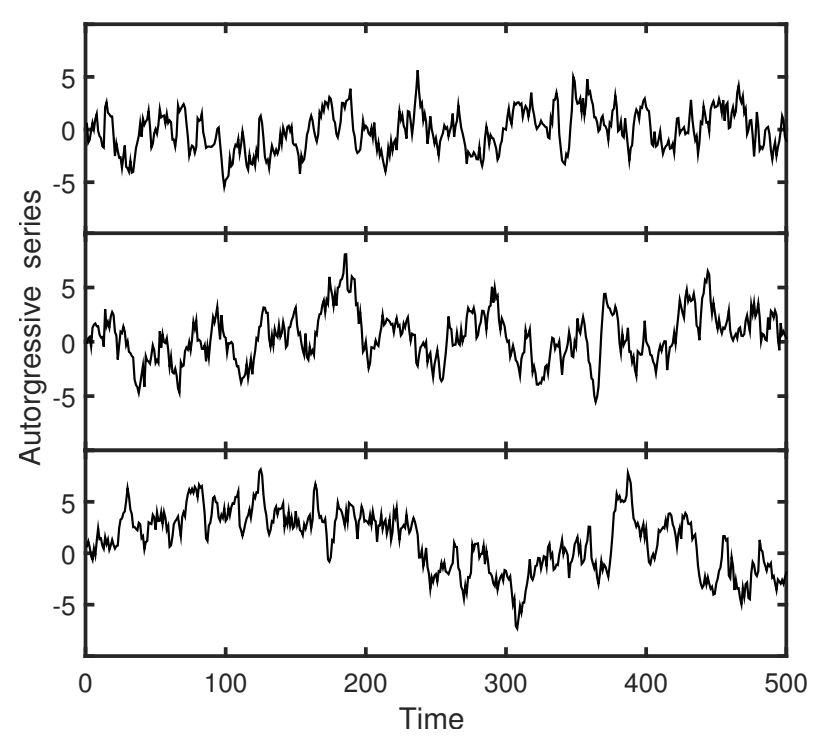

Fig. 5. Three AR time series with respective coefficients $\alpha=0.85$ (top), $\alpha=0.9$ (middle), and $\alpha=0.95$ (bottom).

of the PSD of the accreting stars change with time, while their shapes remain unchanged.

\section{Discussion}

An overview, and expansion, of the material in the previous section follows:

(i) Light curves are interpreted as being due to stochastic processes. If the flux switches from constant mean level $\mu_{1}$ to level $\mu_{2}$, then the ratio of first to second standard deviations will be $\mu_{1} / \mu_{2}$, i.e. the coefficient of variation will remain unchanged.

(ii) If the function $g(t)$ in (2) is deterministic, for example a loworder polynomial, then

$$
\begin{aligned}
E[X(t)] & =g(t) E[z(t)] \\
\operatorname{var}[X(t)] & =g^{2}(t) \operatorname{var}[z(t)] \\
C V(X) & =\sqrt{\operatorname{var}[X(t)]} / E[X(t)] \\
& =\sqrt{\operatorname{var}[z(t)]} / E[z(t)] \\
& \equiv C V(z),
\end{aligned}
$$

i.e. the coefficients of variation of the processes $X(t)$ and $z(t)$ are identical. This means that

$\operatorname{rms}[X(t)]=C V \operatorname{mean}[X(t)]$

at each timepoint $t$, where $C V$ is constant.

(iii) If the flux level is modulated slowly with time by a random trend, as in Figs. 1 and 3, the situation is unchanged, provided the light curve sections which are compared are short compared to the timescale of the trend. For a light curve section centred on time $t=t_{0}$,

$$
\begin{aligned}
E[X(t)] & \approx g\left(t_{0}\right) E[z(t)] \\
\operatorname{var}[X(t)] & \approx g^{2}\left(t_{0}\right) \operatorname{var}[z(t)] \\
C V(X) & \approx C V(z) .
\end{aligned}
$$

(iv) If measurement errors are negligible, then clearly a linear rms-mean relation should have zero intercept. This is exactly equivalent to a constant coefficient of variation. In the presence of measurement errors such that $Y=X+e$,

$\operatorname{var}(Y)=\sigma_{e}^{2}+\operatorname{var}(X)=\sigma_{e}^{2}+(C V)^{2}(E Y)^{2}$ and the intercept will be non-zero. Provided measurement errors and the process $X$ are uncorrelated, and $\sigma_{e}^{2} \ll \operatorname{var}(X)$,

$$
\begin{aligned}
\operatorname{rms}(Y) & \approx\left[1+\frac{1}{2} \sigma_{e}^{2} / \operatorname{var} X\right] \operatorname{rms}(X) \\
& =C V(X)\left[1+\frac{1}{2} \sigma_{e}^{2} / \operatorname{var} X\right] E(Y) .
\end{aligned}
$$

(v) If the stochastic process is strongly autocorrelated, its intrinsic rms will be underestimated over short sections of the light curve. However, provided long enough stretches of the light curve are used, the expected rms-mean relations are recovered.

\section{Comparison to Zamanov et al. (2016)}

Zamanov et al. (2016) obtained several photometric runs on each of nine systems, namely seven cataclysmic variables and two symbiotic stars. In total, 204 light curves were collected. Plotted in a $\log (\mathrm{rms})$ - $\log$ (mean flux) diagram, all but seven points (corresponding to the same star) lie along a well-defined straight line with a slope of 0.994 (standard error 0.042). This is exactly what is expected for constant coefficients of variation:

$\log \sigma=\log \mu+\log C V_{0}$.

Although Eq. (4) could have been anticipated on the basis of (1) for a single object, it is remarkable that Zamanov et al. (2016) obtained such closely similar coefficients of variation $(0.086 \pm$ 0.011 ) for all their objects. This may have something to do with the fact that the accreting stars in all the binary systems they observed were white dwarfs.

\section{Conclusions}

It was demonstrated in Sect. 2 that linear rms-mean flux relations are obtained if a stochastic sequence is subject to simple scaling as in Eq. (2). In that equation, $z(t)$ is an arbitrary stochastic process, and $g(t)$ is a slowly varying modulating function. The physics underlying $z(t)$ and $g(t)$ does not have to be coupled: a constant coefficient of variation of their product $X(t)$ will result provided $g(t)$ is smooth. A prime candidate for the scaling function $g(t)$ is small amplitude variability in the supply of accreting material onto the surface of the accreting object (e.g. Bruch 1992). The only requirement is that the variability timescale be long compared to that of the stochastic process $z(t)$ giving rise to the observed light curves.

Acknowledgements. This work was partially supported by a grant from the South African National Research Foundation. A lunchtime conversation with Prof. Brian Warner stimulated this Letter.

\section{References}

Bruch, A. 1992, A\&A, 266, 237

Chatfield, C. 2003, The Analysis of Time Series: an Introduction 6th edn. (London: Chapman \& Hall/CRC Press)

Gleissner, T., Wilms, J., Pottschmidt, K., et al. 2004, A\&A, 414, 1091

Uttley, P., \& McHardy, I. M. 2001, MNRAS, 323, L26

Uttley, P., \& McHardy, I. M., Vaughan, S. 2005, MNRAS, 359, 345

Van de Sande, M., Scaringi, S., \& Knigge, C. 2015, MNRAS, 448, 2430

Zamanov, R. K., Boeva, S., Latev, G., et al. 2016, MNRAS, 457, L10 